\title{
Globe
}

Revue internationale d'études québécoises

\section{Crise de croissance de l'institution théâtrale québécoise}

\section{Sylvain Schryburt}

Volume 11, numéro 2, 2008

URI : https://id.erudit.org/iderudit/1000526ar

DOI : https://doi.org/10.7202/1000526ar

Aller au sommaire du numéro

Éditeur(s)

Globe, Revue internationale d'études québécoises

ISSN

1481-5869 (imprimé)

1923-8231 (numérique)

Découvrir la revue

Citer cet article

Schryburt, S. (2008). Crise de croissance de l'institution théâtrale québécoise. Globe, 11(2), 159-167. https://doi.org/10.7202/1000526ar d'utilisation que vous pouvez consulter en ligne.

https://apropos.erudit.org/fr/usagers/politique-dutilisation/ 


\title{
PERSPECTIVE
}

\section{CRISE DE CROISSANCE DE L'INSTITUTION THEÂTRALE QUEBECOISE}

\author{
SYLVAIN SCHRYBURT \\ Université d'Ottawa
}

Du 17 au 20 octobre 2007 ont eu lieu, à la Maison Théâtre de Montréal, les Seconds États généraux du théâtre professionnel québécois. Organisé sous l'égide du Conseil québécois du théâtre (CQT), l'événement a rassemblé quelque cinq cents membres de la communauté artistique, incluant des représentants de tous les principaux organismes liés de près ou de loin au théâtre: syndicats des professionnels de la scène, associations de producteurs et autres. Afin de mieux comprendre les raisons qui ont motivé la tenue de cette rencontre des forces vives du théâtre québécois, il importe de remonter quelques années auparavant et de rappeler le climat de crise qui planait alors sur l'ensemble du milieu théâtral.

Entre 2003 et 2005 , en effet, une série de nouvelles inquiétantes a indiqué on ne peut plus clairement que le modèle québécois du thêâtre entrait en état de crise. Au cours de cette période, la presse a ainsi fait état de coupures significatives dans le budget du Festival de théâtre des Amériques de Montréal et du Carrefour international de théâtre de Québec (coupures 
de $700000 \$$ et de $313000 \$$ respectivement ${ }^{1}$ ), de la faillite appréhendée du Thêatre du Rideau Vert - la doyenne des compagnies québécoises - ainsi que de la vétusté des locaux montréalais du Conservatoire d'art dramatique, mais aussi du Thêtre de Quat'Sous. On parlait également de la menace d'un boycott des activités parascolaires par les membres de la Fédération des syndicats de l'enseignement, un moyen de pression visant le gouvernement du Québec mais qui aurait surtout affecté les compagnies de thêâtre jeune public, qui d'ailleurs se remettaient à peine de boycotts semblables tenus en 2000 et en 2002. Il était aussi question du conflit de travail opposant l'Union des artistes (UDA), syndicat des acteurs, aux Théâtres associés inc. (TAI), l'association des plus importants producteurs de spectacles francophones. Résolu in extremis le 17 avril 2005, ce conflit a sérieusement menacé la saison thêatrale 2005-2006. Pour achever ce portrait morose, et bien que la presse d'alors en a peu fait état, mentionnons enfin que l'insatisfaction grandissait chez les compagnies de la relève, dont les activités étaient et sont toujours menacées par un soutien déficient de la part des conseils des arts du Québec et du Canada. Bref, c'est dans ce contexte que la nécessité de faire le point sur l'état du théâtre au Québec s'est rapidement imposée comme une évidence.

Le mot d'ordre fut officiellement lancé, le 12 mai 2005, à l'occasion d'un colloque au titre prémonitoire, Le théâtre à tout prix, organisé par le CQT, qui tenait aussi son assemblée générale le soir même. Lors des plénières de clôture, Pierre MacDuff, directeur général de la compagnie Les Deux Mondes et membre du comité organisateur des Premiers États généraux du théâtre en 1981, proposa à l'assemblée de confier au CQT le mandat de préparer la tenue de nouveaux États généraux pour l'année 2007. La proposition fut adoptée à la majorité et la tâche d'organiser l'événement confiée au metteur en scène Martin Faucher, fraîchement nommé à la présidence du Conseil.

Dans les dix-huit mois qui suivirent, le grand défi du CQT consista à réunir l'ensemble des intervenants du milieu afin de présenter un front commun face aux gouvernements lorsque viendrait le temps de donner suite aux décisions prises en plénière ${ }^{2}$. Ce front commun, souhaité par les quelques artistes et journalistes à avoir pris publiquement position en faveur de la tenue de l'événement, moi-même y compris, a bel et bien été constitué,

$$
+\div
$$

1. Voir Isabelle PORTER, "Québec - Le Carrefour de thêatre crie famine", Le Devorr, 17 juin 2005.

2. Il peut être utile de rappeler ici le coup de thêâtre qu'avait constitué, le 6 novembre 1981 , à quelques heures seulement de l'ouverture des Premiers Étars généraux, le retrait de l'Associarion des direcreurs de théâtre (ADT, précurseur de TAI), une situation que personne ne voulait voir se répeter. 
mais il s'est avéré, en dernière analyse, le talon d'Achille de ces États généraux. Comment, en effet, concilier les intérêts spécifiques, pour ne pas dire corporatistes, de trois syndicats ${ }^{3}$, de quatre associations de producteurs ${ }^{4}$ et des nombreux autres organismes de théâtre? Comment satisfaire tout à la fois les doléances des dramaturges, des acteurs, des metteurs en scène, des directeurs de compagnie et autres professionnels de la scène? Comment obrenir un consensus autour des solutions à apporter à des enjeux aussi variés que la diffusion du théâtre québécois à l'échelle nationale et internationale, le développement de public, l'accessibilité des salles de spectacle pour les compagnies de la relève ou encore les difficultés propres au théâtre en région, au théâtre jeune public, et j'en passe? Enfin, comment prendre acte, dans un ensemble cohérent de propositions, et sans s'aliéner quiconque, des besoins particuliers des compagnies institutionnelles, des compagnies intermédiaires financées au "fonctionnement" ainsi que des compagnies dites de la relève, financées "au projet" ? La réponse est simple: il faut d'abord éviter que soient soulevées des questions épineuses. Il faut ensuite formuler des propositions qui feront d'office consensus, c'est-à-dire qui remettent la responsabilité d'agir sur les épaules des gouvernements en réclamant, par-dessus tout, un investissement financier massif de leur part. À l'évidence, telle fut la stratégie adoptée.

\section{DES ETATS GÉNERRAUX CONSENSUELS}

Des 59 propositions adoptées lors des plénières des États généraux d'octobre 2007, la plupart à l'unanimité et sans l'ombre d'un débat, 44 exigent d'une manière ou d'une autre un financement accru du théâtre par l'un ou

$$
++4
$$

3. La doyenne UDA (1937), à laquelle se sont ajoutées plus récemment l'Association des professionnels des arts de la scène du Québec (APASQ, 1984) et l'Association québécoise des auteurs dramatıques (AQAD, 1990).

4. Les Theâtres associés ınc. (TAI, 1985), les Theâtres unıs enfance jeunesse inc. (TUEJ, 1985), l'Association des producteurs de chéâtre privé (APTP, 1986) ec l'Association des compagnies de theâtre (ACT, 1989).

5. Une compagnie est qualifiée d'insttutuonnelle lorsqu'elle dispose d'une salle permanente où elle programme des saisons de chéatre comportant environ quacre à cinq spectacles, parfois en coproduction, plus rarement comme simple diffuseur. Étant donné les coùcs d'opération eleves de ce type de compagnies, elles disposent d'ordinaire de subventions beaucoup plus importantes. Dans cetce catégorie figurent le Theâtre du Nouveau Monde, le Thêâtre du Rideau Vert, l'Espace GO, le Thêâtre de la Bordée, le Théâtre Denise-Pelletier, le Theâtre d'Aujourd'hui ou encore le Théâtre de Quat'Sous, plus perit. Aux yeux du Conseil des arts du Canada (CAC) ec du Conseil des arts et des lettres du Québec (CALQ), les compagnies instıturionnelles sont soutenues au fonctionnement, c'est-à-dire de façon pluriannuelle, ce qui permer de planifier une gescion à long terme de leurs activités. Dans les faits, cependant, les subventions au fonctionnement sont aussi l'apanage des compagnies de statut intermédıaıre, dont certaines sont toutefoss soutenues de façon annuelle. J'emploie néanmoins l'expression de compagnies au « fonctionnement » pour désigner ces compagnies intermédiaires qui, contrairement à leurs aînées institutionnelles, ne possèdent pas de lieu qui leur appartienne en propre et proposent seulement un ou deux spectacles par saison. Entrent dans cette catégories des compagnies aussi variées que Sibyllines, le Theâtre UBU, le Thêâtre de l'CEal, le Théâtre Il va sans dire ou le Theâtre de Quarrier. Enfin, la catégorie des compagnies financtes par "projet " constitue le dernier échelon de cette pyramıde à trois paliers. Ici, les programmes soutiennent un spectacle en particulier, sans aucune récurrence. 
l'autre des paliers de gouvernement. Je pense ici aux propositions recommandant la bonification des bourses et des programmes existants (propositions $7 \mathrm{~A}, 28$ [reformulée en plénière], 38, 46, 50 et autres ${ }^{6}$ ), la création de nouveaux programmes et bourses (propositions 12, 43.1, 43.2, 47, 60, 48A, etc.), l'assouplissement des critères d'admissibilité à certains programmes, l'imposition d'une nouvelle taxe pour financer la culture, la mise sur pied de divers incitatifs fiscaux, etc. $\mathrm{Si}$ plusieurs de ces propositions impliqueraient des investissements minimes et pourraient aisément être mises en place, d'autres, en revanche, engageraient d'importants montants récurrents, telles la proposition de développer un réseau de petites salles dans les grands centres urbains et en région (proposition 13), celle de rendre obligatoire la fréquentation du théâtre professionnel par "tous les élèves du Québec" (proposition 51), ou encore de créer deux nouveaux "ensembles théâtraux d'envergure" (proposition 73), l'un à Montréal et l'autre à Québec, c'est-à-dire des compagnies permanentes, comme l'avaient demandé les fondateurs du Thêâtre du Nouveau Monde dès les années 1950.

Mais au-delà du bien-fondé de chacune de ces propositions, que l'on s'est d'ailleurs bien gardé de chiffrer, il faut retenir que l'ensemble des intervenants du milieu a pu y trouver son compte puisque ces nouveaux programmes ou bonifications, s'ils étaient effectivement mis sur pied, toucheraient à la fois les auteurs, les acteurs et les autres professionnels de la scène, tout comme les diffuseurs et les compagnies, grandes et petites. En somme, la vision de l'avenir qui se dégage de cette liste d'épicerie se résume, ou presque, à ceci: plus d'argent pour tous et une parfaite égalité dans la répartition de cette manne, puisque aucun secteur prioritaire n’a été désigné aux instances gouvernementales. Dans ces conditions, doit-on se surprendre d'avoir obtenu un consensus de tous les intervenants du milieu?

L'occasion était pourtant belle de faire des choix d'avenir qui sortent un tant soit peu des sentiers battus. Car la crise actuelle qui frappe le milieu théâtral est en fait une véritable crise de croissance: de plus en plus de joueurs réclament leur part des fonds alloués par les divers paliers de gouvernement, même si les subventions stagnent ou régressent depuis 2004. Cela alors que, selon l'Observatoire de la culture et des communications du Québec, le taux d'occupation des salles est au beau fixe depuis lors à environ $73 \%$ (un peu moins de $63 \%$ si l'on compte uniquement les entrées

$$
+4
$$

6. J'inclus dans cecte carégorıe les propositıons qui demandent la a reconnaissance " par les pouvoirs publics de l'importance de certaıns secteurs d'activité et leur « financement adéquat". 
payantes) ${ }^{7}$. Or, qu'on le veuille ou non, il n'y a que trois avenues pour rétablir un équilibre entre l'offre des subventions et les demandes du milieu: augmenter la première, réduire les secondes ou jouer sur les deux plans à la fois. Admettons un instant que les propositions des derniers États généraux soient toutes mises en pratique. Nous verrions alors la création de deux nouvelles institutions permanentes (avec leurs propre théâtre?), la mise en place d'un réseau de nouvelles salles sur l'ensemble du territoire, la stabilisation de l'ensemble des compagnies existantes via la bonification de leurs subventions, l'atrribution de nouvelles subventions au "fonctionnement" pour un nombre indéterminé de compagnies actuellement financées au "projet", l'accès au financement par "projet" des troupes qui, pour l'instant, passent sous les écrans radar du Conseil des arts du Canada (CAC) et du Conseil des arts et des lettres du Québec (CALQ), et j'en passe. Il s'agirait, en somme, d'une croissance généralisée sur tous les fronts.

Pour séduisante que puisse être cette vision de l'avenir, elle m'apparaît de courte vue. En effet, il existe à l'heure actuelle pas moins de six écoles francophones qui forment des professionnels du thêâtre sur l'ensemble du territoire québécois. Cela signifie qu'année après année, près de soixantedix acteurs rejoignent les rangs du milieu théâtral, sans compter les metteurs en scène, les auteurs, les scénographes et les autres professionnels de la scène à qui ces écoles proposent aussi des programmes spécifiques. Or, pour seulement espérer pratiquer leur art, plusieurs de ces finissants n'ont d'autre choix que de former de nouvelles compagnies, condition d'admissibilité sine qua non aux programmes de subvention par projet. Certes, on ne peut pas reprocher aux jeunes artistes de vouloir exercer leur art et d'en vivre. Mais dans les circonstances, on ne doit pas s'étonner non plus qu'il y ait engorgement du système, que les fonds alloués soient nettement inférieurs à la demande et que cet écart aille croissant. Et même en admettant que l'on parvienne à débloquer considérablement l'échelon inférieur du système de financement, il ne fait nul doute que ces nouvelles subventions attribuées au "projet" trouveront rapidement preneurs, avec la hausse que cela impliquerait sur la demande en petites salles de spectacle. Pourtant, malgré ce déséquilibre, à nul moment lors de ces États généraux n'a-t-il été question de la formation, et encore moins - sujet tabou par excellence - du nombre même des écoles professionnelles.

$$
+\div
$$

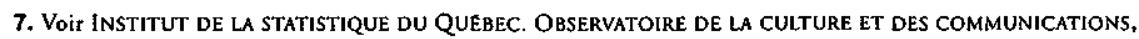
Statstiques en bref, $n^{\circ} 41$, seprembre 2008, p. 6 . 
Mais laissons de côté ce problème épineux pour examiner ce qui se produirait ensuite. On ne peut faire partie, sa vie durant, de la relève et cette dernière aspirera, en toute légitimité, à accéder un jour aux subventions au "fonctionnement". Or, j'ai peine à croire que cette relève remplacera à moyen terme les compagnies qui, aujourd'hui, obtiennent ce type de financement, et qu'ainsi se créera un équilibre, naturel pour ainsi dire, dans la succession des générations d'artistes. En effet, dans les dix à vingt ans qui viennent, la question de la succession se posera avec insistance au sein de nombreuses compagnies fondées et dirigées par des baby-boomers, plus vraisemblablement chez celles dont le directeur n'est pas un metteur en scène. Ces derniers souhaiteront-ils confier les rênes de leur organisme à la génération suivante, qui pourrait ainsi profiter des avantages que conferent l'ancienneté et l'institutionnalisation lorsqu'il s'agit de demander les subventions du CAC ou du CALQ? On peut croire que c'est la voie que choisiront plusieurs d'entre eux. Peut-on en effet consacrer trente, quarante ou cinquante ans de sa vie à une compagnie, développer des réseaux locaux et internationaux, s'attacher une petite poignée d'employés et accepter sereinement de mettre la clé dans la porte au jour de la retraite? On peut en douter. Dès lors, une question importante se pose: combien de ces compagnies intermédiaires sont appelées, dans un futur indéterminé, à devenir des institutions pérennes dont le statut est quasi intouchable dans le modèle du thêâtre québécois actuel? Combien de joueurs, anciens et nouveaux, se feront la lutte à ce niveau, alors qu'au bas de l'échelle on formera toujours autant de jeunes professionnels? Bref, il y a fort à parier que de nouveaux États généraux devront être convoqués, dans vingt ou vingt-cinq ans, pour faire face une fois de plus au même cul-de-sac persistant, à une semblable crise de croissance: ressources insuffisantes, stagnation des compagnies, portes fermées pour la relève, etc. Tel était le cas en 1981, tel est le cas actuellement et tel sera toujours le cas demain si l'on s'abstient, aujourd'hui, de faire des choix, d'imposer des priorites. Et l'une d'entre elles me semble être de réfléchir, dans un premier temps, au rôle des écoles de formation dans l'écologie du système actuel, et, dans un second temps, de prendre résolument le parti de la jeunesse.

\section{FAIRE DE LA JEUNESSE}

\section{ET DU MÉRITE ARTISTIQUE UNE PRIORITÉ}

L'incapacité du milieu théâtral à identifier des secteurs d'intervention prioritaires offre un contraste flagrant avec les États généraux de 1981, où l'on avait justement fait le pari de miser sur la jeunesse. En 1981, même si les besoins de tout un chacun avaient été également réaffirmés, un constat clair 
avait émergé avant même la tenue de l'événement: à l'époque, les onze compagnies institutionnelles se partageaient $70 \%$ des principales subventions accordées par les trois paliers de gouvernement, tandis que les $30 \%$ restants étaient divisés entre 90 compagnies - toutes jeunes, il va de soi. En conséquence, l'un des principaux effets de ces Premiers États généraux a été de remplacer l'ancien statut de compagnies "institurionnelles" par celui de compagnies soutenues au "fonctionnement" annuel ou pluriannuel afin de faire entrer dans cette nouvelle catégorie un nombre croissant de compagnies fondées, pour la plupart, durant les décennies 1970 et 1980, et qui demeurent pour l'essentiel actives à ce jour. Pour accommoder les autres joueurs du milieu théâtral, on créa également le statut de "subventions au projet", des fonds distribués à la pièce, c'est le cas de le dire, sans récurrence aucune.

Or, les jeunes compagnies d'aujourd'hui, celles qui ont été fondées durant les décennies 1990 et 2000 , sont à leur tour dans une situation semblable à celle où se trouvaient leurs aînées vingt-cinq ans plus tôt. Elles fonctionnent pour la plupart au projet, représentent $47 \%$ des compagnies soutenues par le CAC ( 51 bourses sur 107 ) et $36 \%$ des compagnies soutenues par le CALQ (36 sur 99) et doivent se partager, selon les statistiques de 2006-2007 compilées par le CQT ${ }^{8}$, 9,5\% des montants versés par les principaux programmes du CAC et $5 \%$ de ceux du CALQ. Et ces chiffres ne disent rien, bien sûr, des autres jeunes compagnies qui ne demandent aucune forme de soutien, qui ont vu leurs demandes rejetées ou qui organisent des soupers bénéfices pour financer leurs activités. Prendre le parti de la jeunesse aurait consisté à prendre acte de cet état de fait. Dans les circonstances actuelles, parce qu'elles sont noyées dans une logique du tout pour tous, on est en droit de se demander quel poids auront des demandes telles que la création d'un lieu de diffusion consacré aux compagnies de la relève et, surtout, l'augmentation des budgets versés au "projet»? Prendre le parti de la jeunesse consisterait aussi à accepter que des compagnies puissent disparaître ou ne pas survivre au départ de leur fondateur, et à reconnaître qu'on ne soutient pas les organismes, mais bien ceux qui les animent.

Dans le même ordre d'idées, mais en songeant cette fois aux compagnies financées au "fonctionnement", il m’apparaît impératif de réformer des modes d'attribution de subventions qui favorisent des

$+\div$

8. Pourcentage compilé à partir de staristiques fournies par le CQT (vorr pages 55 er 57 du "Cahier du participant* distribué lors des États généraux et disponible en ligne sur le sire du Conseil: http://www.cqu.ca). Il est à nocer que ces compilations sont elles-mêmes basées sur des données fournies par l'Observaroire de la culture er des communications du Québec. 
compagnies établies de longue date, même si leur productions sont pour le moins conventionnelles, sinon incolores. Durant les États généraux, une seule résolution a été formulée en ce sens, la rafraichissante proposition 33 que je me suis étonné de voir adoptée à l'unanimité et sans débat tant elle m'est apparue quasi révolutionnaire dans ce contexte: "que le montant des sommes octroyées par le Conseil des arts du Canada aux compagnies soutenues au fonctionnement soit déterminé sur la base du mérite artistique sans l'imposition d'aucun plafond de pourcentage, à la hausse ou à la baisse".

Cette proposition détonne non seulement parce qu'elle est la seule qui fasse état du "mérite artistique», mais aussi parce que son long préambule met le doigt sur l'un des vices du système actuel qui favorise indûment les compagnies établies aux dépens des nouvelles venues plus novatrices, et nourrit par la même occasion un écart sans cesse grandissant entre le soutien accordé aux unes et aux autres. En effet, à l'heure actuelle, les augmentations allouées par le CAC représentent un pourcentage donné de la subvention précédente, ce qui avantage évidemment les compagnies actives depuis plusieurs décennies et accroît les inégalités entre les joueurs du milieu, sans tenir compte de l'excellence et surtout du risque, artistique comme financier, que peut représenter la production de spectacles de recherche ou aux visées plus exigeantes. Que l'État investisse davantage dans le théâtre, soit, mais pourquoi ces nouvelles sources ne devraient-elles pas soutenir en priorité les besoins de créateurs aux univers singuliers et novateurs plutôt que l'industrie de la culture, le théâtre de divertissement ou le thêâtre de loisir qui s'avèrent par nature plus rentables?

Entendons-nous bien: je suis de ceux qui sont parfaitement convaincus que le théâtre, comme d'ailleurs les autres secteurs culturels - à commencer par celui de la danse -, souffre d'un sous financement chronique. En cela, je souscris et j'endosse les thèses fondamentales du Mouvement pour les arts et les lettres (MAL) et partage la plupart de ses revendications, à commencer par l'augmentation substantielle des budgets alloués aux arts et l'indexation de ceux-ci au coût de la vie. Seulement, on l'aura compris, je ne suis pas de ceux qui croient qu'une injection massive d'argent pour tous soit la panacée à la présente crise, ni de ceux qui pensent qu'il n'y aura jamais trop de théâtre, de danse, de livres, bref de culture au Québec. Ce genre de posture angélique qui fétichise la "création" à tout prix sans jamais distinguer entre le divertissement et l'art à risque, mais en posant comme un droit inaliénable le soutien de toutes les formes d'expressions artistiques, rend d'autant moins crédible le discours du milieu face aux décideurs publics qui, eux, doivent composer avec le réel. Il me semble plus que jamais essentiel de rejeter cette 
posture du "tout pour tous", quitte à mettre en jeu la belle unanimité du milieu. Il me semble plus que jamais essentiel de prendre le parti de l'art contre le ronron de l'industrie culturelle, particulièrement dans les institutions actuelles, mais aussi d'admettre qu'il existe bel et bien un déséquilibre entre les générations. 